\title{
Myocardial impact and cardioprotective effects of apelin-13 and a c-terminal-modified analog during LPS and CLP experimental sepsis
}

O Lesur

From ESICM LIVES 2015

Berlin, Germany. 3-7 October 2015

\section{Introduction}

Apelin-13 (APL-13) is a member of an endogenous peptide's family (APLs) with powerful inotropic and cardioprotective properties. APLs bind to the dedicated receptor APJ-R, a member of the $G$ protein-coupled receptor superfamily, all being widely expressed in the cardiovascular system. We have already shown that APL-13 infusion, was protective against LPS-induced myocardial dysfunction and death vs. dobutamine [1]. Furthermore, we have shown that, C-terminal Phe(13) modification of APL-13 by unnatural amino acids can change ligand binding and APJ-R signaling [2].

\section{Objectives}

Understanding the beneficial impact of APL-13 on LPSinduced myocardial injury vs. dobutamine, and assessing functional and biological effects of a new selected linear APL-13 analog with enhanced affinity, and their impact in the context of sepsis.

\section{Methods}

Myocardial dysfunction was induced by intra-peritoneal injection of LPS (E. Coli 055:B5, $10 \mathrm{mg} / \mathrm{kg}$ ) or Cecal Ligature and Puncture (CLP) in male Sprague-Dawley rats. Myocardial injury was biologically evaluated by analyzing of different cellular pathway of apoptosis and inflammation by Western blot. Myocardial function was assessed ex-vivo by Langendorff and in vivo by echocardiography by comparing APL-13 to $\operatorname{Tyr}(\mathrm{Obn})$. Tyr(OBn) (13) substitution led to a 60 -fold increase in binding affinity vs. APL-13 [2].

\section{Results}

LPS-challenged rats treated with APL-13 exhibited a clear reduction of both apoptosis (cleaved caspase-3, BAX/BCL-2 ratio) and inflammation (iNOS and MIF) markers, with significant alterations in the Akt/GSK3b/ $\mathrm{mTor}$ and P38/Erk pathways underscoring the cardioprotective effect of APLs. Organic Langendorff assays confirmed cellular data [2] in that enhanced affinity confers to $\mathrm{Tyr}(\mathrm{Obn})$ analog a more effective and potent inotropic activity than APL-13, as shown by the increased left ventricular developed pressure (LVDP) (\% baseline, 1pM : APL-13, $8 \pm 13$ vs. Tyr(Obn), $60 \pm$ $15 ; \mathrm{p}<0.05)$, (30pM : APL-13, $124 \pm 25$ vs. Tyr(Obn), $372 \pm 106 ; \mathrm{p}<0.05)$. APLs sensibility was increased in $8 \mathrm{~h}$ CLP-challenged hearts, as it was in 24h LPS-challenged hearts [1], suggesting upregulation of the myocardial apelinergic pathway during polymicrobial sepsis. Indeed, CLP model of sepsis was characterized at $8 \mathrm{~h}$ by a reduced cardiac output (Sham, $170 \pm 3$ vs. CLP, $99 \pm 5 \mathrm{ml} / \mathrm{min}, \mathrm{p}<$ 0.05 ) with an increased parietal thickness (Sham, $0.15 \pm$ 0.002 vs. CLP, $0.23 \pm 0.003 \mathrm{~cm}, \mathrm{p}<0.05)$ in vivo.

\section{Conclusions}

APLs are new safer supporting drugs in sepsis. Chemical modifications can optimize the inotropic potency of APLs opening a novel field of therapeutic opportunities. Ongoing works are to evaluate the functional effect of these new analogs in vivo, with Pressure-Volume curve device, and to further test their comparative cardioprotective potential during experimental CLP sepsis.

\section{Grant Acknowledgment}

nHSF, Merck. 


\section{References}

1. Lesur O, Chagnon F, Murza A, Sarret P, Marsault E, Salvail D: Apelin is cardioprotective and life-saving over dobutamine in a murine model of endotoxin-induced myocardial dysfunction. Intensive Care Med Exp 2014, 2(Suppl 1):P11, 0100

2. Murza A, Besserer-Offroy É, Côté J, Bérubé P, Longpré JM, Dumaine R, et al: C-Terminal modifications of apelin-13 significantly change ligand binding, receptor signaling, and hypotensive action. J Med Chem 2015, 58(5):2431-2440.

doi:10.1186/2197-425X-3-S1-A436

Cite this article as: Lesur: Myocardial impact and cardioprotective effects of apelin-13 and a c-terminal-modified analog during LPS and CLP experimental sepsis. Intensive Care Medicine Experimental 2015 3(Suppl 1):A436.

\section{Submit your manuscript to a SpringerOpen ${ }^{\mathcal{O}}$ journal and benefit from:}

- Convenient online submission

- Rigorous peer review

- Immediate publication on acceptance

- Open access: articles freely available online

- High visibility within the field

- Retaining the copyright to your article 